\title{
Efecto del uso intraoperatorio de corticoides en dosis bajas en pacientes sometidos a resección hepática mayor con maniobra de Pringle
}

\author{
Gonzalo Yáñez C. ${ }^{1}$, Constanza Godoy S. ${ }^{2}$, Dahiana Pulgar B. ${ }^{3}$, Carlos Maldonado L. ${ }^{2}$, \\ Juan Francisco Guerra C. ${ }^{2}$, Nicolás Jarufe C. ${ }^{2}$, Eduardo Briceño V. ${ }^{2}$ y Jorge Martínez C. ${ }^{2}$
}

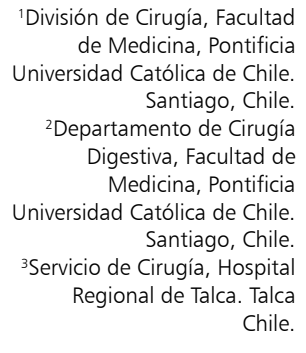

Conflictos de interés: No hay.

Recibido el 13 de octubre de 2017, aceptado para publicación el 19 de diciembre de 2017.

Correspondencia a: Dr. Jorge Martínez C. jamartin@med.puc.cl
Effects of low dose corticosteroids in patients undergoing major hepatic resection with Pringle maneuver

Introduction: Liver resections may be associated with high morbidity and mortality due to intraoperative bleeding. Pringle maneuver reduces this complication at the expense of ischemia-reperfusion injury. Current strategies to minimize reperfusion injury include the use of perioperative corticosteroids. Objective: To assess the use of methylprednisolone in low doses $(<500 \mathrm{mg})$ in patients submitted to major hepatic resection under Pringle maneuver in the incidence of ischemia-reperfusion injury, peri-operative morbidity, and mortality. Material and Methods: Retrospective study from the liver resections database undertaken between the years 2000-2015 in our center. One hundred and seventy-one major liver resections were done, in 62 under Pringle maneuver. Two groups were established: (A) Patients administered methylprednisolone immediately before Pringle maneuver $(n=27)$ and $(B)$ those without steroid $(n=35)$. We assessed ischemia-reperfusion injury by measuring liver tests on days 1, 3 and 5 . Results: Patients in group A had longer ischemia time $(43 \pm 3.3 v s .27 \pm 2.1 \mathrm{~min}, \mathrm{p}<0.05)$ than those of group $\mathrm{B}$, and significantly lower elevation of serum phosphatase alkaline and bilirubin on days 1 and five post-hepatectomy. We did not observe any difference in bleeding magnitude, and there were no differences in morbidity or mortality. Conclusions: The use of low doses of methylprednisolone seems to diminish the impact of ischemia-reperfusion injury related to major hepatic resection under Pringle maneuver avoiding the adverse side effects of high dose steroid.

Key words: hepatectomy; corticosteroids; reperfusion injury.

\section{Resumen}

Introducción: Las resecciones hepáticas mayores pueden presentar una alta morbimortalidad en relación al sangrado intraoperatorio. La utilización de la maniobra de Pringle permite disminuir esta complicación a costa de un daño por isquemia-reperfusión. Una estrategia para minimizarla es el uso de corticoides perioperatorios. Objetivo: Evaluar el uso de metilprednisolona en dosis bajas $(<500 \mathrm{mg})$ en pacientes sometidos a resección hepática mayor con maniobra de Pringle en la incidencia de daño por isquemiareperfusión, morbilidad y mortalidad perioperatoria. Material y Métodos: Estudio retrospectivo utilizando la base de datos de hepatectomías entre los años 2000 y 2015. De un total de 171 resecciones hepáticas mayores, 62 utilizaron clampeo vascular. Se establecieron dos grupos: (A) aquellos que recibieron metilprednisolona inmediatamente previo al clampeo $(\mathrm{n}=27)$ y $(\mathrm{B})$ pacientes sin metilprednisolona $(\mathrm{n}=$ 35 ). Se evaluó el daño por isquemia-reperfusión por alteración de las pruebas hepáticas en los días 1,3 y 5. Resultados: Los pacientes del grupo A tuvieron mayor tiempo de isquemia $(43+3,3$ vs $27+2,1$ min, $p<0,05$ ) que el grupo $B$, con una significativamente menor elevación de las fosfatasas alcalinas y bilirrubina en los días 1 y 5 poshepatectomía. No se observó diferencias en la magnitud del sangrado y no hubo diferencias en morbimortalidad. Conclusiones: La utilización de dosis bajas de metilprednisolona parece disminuir el impacto del DIR relacionado a la resección hepática bajo clampeo vascular, evitando los efectos adversos de la administración de esteroides en dosis altas.

Palabras clave: hepatectomía; corticoides; daño por isquemia-reperfusión. 


\section{Introducción}

La hepatectomía es un procedimiento quirúrgico indicado en el tratamiento de tumores hepáticos malignos y en otras patologías hepáticas benignas y trauma. La mejoría en la técnica quirúrgica, cuidados perioperatorios y derivación a centros especializados, ha incidido en un aumento del número de pacientes sometidos a esta operación y ha permitido disminuir su morbilidad y mortalidad ${ }^{1}$. Un factor relevante asociado a mortalidad en resecciones hepáticas mayores (RHM) es el sangrado intraoperatorio y la necesidad de transfusiones perioperatorias. Para controlar lo anterior, se ha propuesto la interrupción controlada del flujo sanguíneo hacia el hígado mediante el pinzamiento vascular o maniobra de Pringle (MP) (Figura 1). Esta maniobra, tiene como efecto secundario un daño por isquemia-reperfusión (DIR), lo cual es relevante en hígados previamente dañados, con esteatosis o que han sido sometidos a quimioterapia ya que puede contribuir a una insuficiencia hepática posoperatoria (IHP). Existen varias definiciones de IHP, una de ellas son los criterios "50-50" propuesto por Balzan et $\mathrm{al}^{2}$ que la define como la combinación de TP $<50 \%$ (INR $>1,7)$ y bilirrubina plasmática $>50 \mathrm{umol} / \mathrm{L}(>2,9 \mathrm{mg} / \mathrm{dL})$ al día posoperatorio 5 . Los principales factores de riesgo asociadas a su desarrollo son la hemorragia intraoperatoria, extensión de la resección hepática y la duración de la $\mathrm{MP}^{3}$. Han sido publicadas diversas estrategias para disminuir su impacto entre las que destacan la MP intermitente, el preacondicionamiento isquémico y algunas intervenciones farmacológicas como el uso de metilprednisolona (MPD) en dosis de $500 \mathrm{mg}$ o más. El objetivo de nuestro trabajo es evaluar el uso de esteroides en dosis bajas $(<500 \mathrm{mg})$ en pacientes sometidos a RHM con MP en la incidencia de DIR, morbilidad y mortalidad perioperatoria.

\section{Material y Métodos}

\section{Diseño}

Se realizó un estudio de cohorte retrospectivo utilizando la base de datos de resecciones hepáticas del Hospital Clínico de la Pontificia Universidad Católica de Chile entre enero de 2000 y abril de 2015. Se incluyeron en el estudio pacientes adultos, sometidos a RHM bajo MP. Se excluyeron pacientes sometidos a hepatectomías segmentarias y no anatómicas. Se obtuvieron datos biodemográficos y de riesgo quirúrgico según ASA. La variable de exposición fue la utilización de MPD intravenosa previo al clampeo vascular en dosis bajas (180-240 mg) y la variable resultado, el desarrollo de DIR, valorada

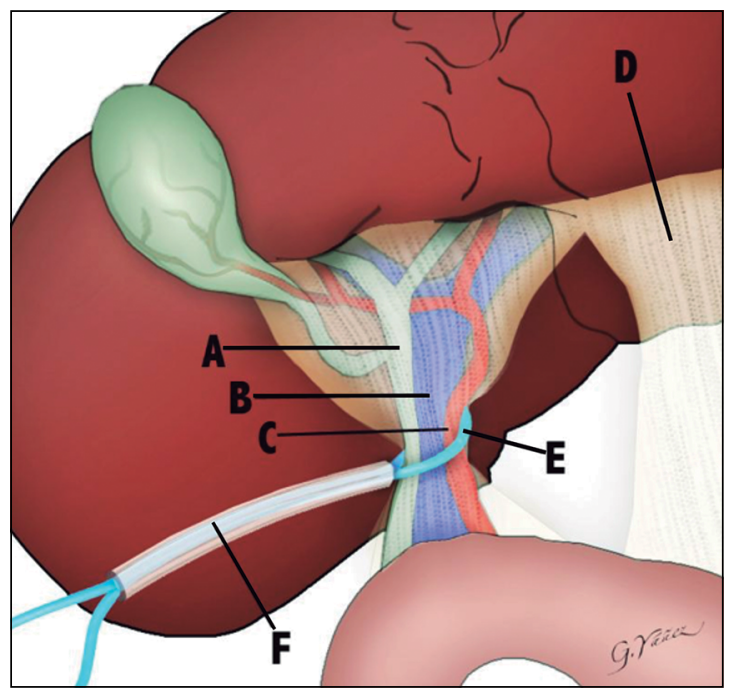

Figura 1. Representación de la Maniobra de Pringle. A: Vía biliar; B: Vena porta; C: Arteria hepática; D: Ligamento hepatoduodenal; E: Cinta ; F: Tubo. por pruebas hepáticas posterior a la intervención. Los pacientes fueron divididos en un grupo (A) a quienes se les administró MPD $(\mathrm{n}=27)$ y un grupo (B) sin el empleo de esteroides $(\mathrm{n}=35)$. Se indicó utilizar esteroides por vía intravenosa a juicio del cirujano en el intraoperatorio de acuerdo a los factores de riesgo presentes. En el posoperatorio se realizó medición plasmática de pruebas hepáticas y coagulación al día 1, 3, 5 y al alta del paciente. Además, se evaluó el alza máxima de aminotransferasas en ambos grupos. Las complicaciones posoperatorias fueron clasificadas según Dindo-Clavien ${ }^{4,5}$.

\section{Análisis estadístico}

Las variables continuas se expresaron como promedio y desviación estándar para datos paramétricos y su comparación se realizó utilizando la $t d e$ student. Los datos no paramétricos se expresaron como medianas y rango, utilizando en su comparación el test de Mann-Whitney. En el caso de variables cualitativas nominales se utilizó el test de $x^{2}$. Las diferencias fueron consideradas significativas con un $\mathrm{p}<0,05$. El análisis estadístico fue realizado utilizando el programa informático SPSS versión 21.

\section{Resultados}

En el período descrito se realizaron 246 hepatectomías, de las cuales 171 correspondieron a RHM $(69,5 \%)$; de ellas 62 bajo MP (36,3\%). La edad media de la población fue de $59 \pm 12$ años, en su mayoría del sexo masculino $(61,3 \%)$ y con riesgo quirúrgico estratificado como ASA I-II (90,3\%). Las indicaciones de resecciones hepáticas más frecuentes fueron metástasis hepática de cáncer colo- 
Tabla 1. Descripción de la población y variables preoperatorias

\begin{tabular}{|lccc|}
\hline & $\begin{array}{c}\text { Grupo A } \\
(\mathbf{n = 2 7})\end{array}$ & $\begin{array}{c}\text { Grupo B } \\
(\mathbf{n = 3 5})\end{array}$ & $\mathbf{p}$ \\
\hline Edad (años)* & $57(24-84)$ & $61(37-80)$ & 0,19 \\
Sexo H/M & $16 / 11$ & $22 / 13$ & 0,773 \\
Comorbilidad general & 14 & 23 & 0,270 \\
ASA & & & 0,867 \\
ASA I & 5 & 6 & \\
ASA II & 20 & 4 & \\
ASA III & 2 & 0 & 0,782 \\
ASA IV-V & 0 & $26(15-95)$ & 0,906 \\
GOT (U/L)** & $29(11-145)$ & $31(10-150)$ & 0,139 \\
GPT (U/L)** & $30(10-475)$ & $64(16-1029)$ & 0,671 \\
GGT (U/L)** & $40(0,0-641)$ & $116,5(58-702)$ & 0,254 \\
FA (U/L)** & $110(52-481)$ & $0,51(0,21-22,75)$ & 0,552 \\
BT (mg/dL)** & $0,59(0,2-16,8)$ & $0,19(0,01-17,89)$ & 0,881 \\
BD (mg/dL)** & $0,19(0-12,9)$ & $86,9(12,1)$ & 0,38 \\
\hline TP (\%)* & $86,1(25,1)$ & 38,2 & \\
\hline Hematocrito (\%) $*$ & $39,2(4,8)$ & & \\
\hline
\end{tabular}

*Valores expresados como medias (DE: desviación estándar); **valores expresados como medianas (rango); H: hombre; M: mujer; ASA: clasificación de la American Society of Anesthesiologists; BT: bilirrubina total; BD: bilirrubina directa; TP: tiempo de protrombina; GOT: transaminasa glutámico oxalacética; GPT: transaminasa glutámico pirúvica; GGT: gamma-glutamil transpeptidasa; FA: fosfatasas alcalinas.

Tabla 2. Descripción de las indicaciones de resección hepática mayor

\begin{tabular}{|lcc|}
\hline & $\begin{array}{c}\text { Grupo A } \\
(\mathbf{n}=\mathbf{2 7})\end{array}$ & $\begin{array}{c}\text { Grupo B } \\
(\mathbf{n}=\mathbf{3 5})\end{array}$ \\
MHCCR & 10 & 15 \\
Colangiocarcinoma & 6 & 7 \\
Hepatocarcinoma & 4 & 7 \\
Hepatolitiasis & 2 & 4 \\
Cáncer de vesícula & 3 & 0 \\
Hemangioma hepático & 1 & 1 \\
Quiste hidatídico & 0 & 1 \\
Hepatoblastoma & 1 & 0 \\
Cistoadenoma hepatobiliar & 0 & 1 \\
\hline MH no CCR no NE & 0 & 3 \\
\hline
\end{tabular}

$\mathrm{p}=0,528$; MHCCR: metástasis hepática cáncer colorrectal; MH no CCR no NE: metástasis hepática no cáncer colorrectal ni neuroendocrino: un paciente con diagnóstico de metástasis hepática de cáncer medular de tiroides, una metástasis hepática de leiosarcoma retroperitoneal y una enfermedad relacionada a IgG. rrectal (MHCCR) en un 40,3\%, colangiocarcinomas (intrahepáticos y tumor de Klatskin) en un $21 \%$, y hepatocarcinomas en un $11,3 \%$. Respecto al tipo de RHM, se realizaron 23 hepatectomías derechas (HD, 37,1\%), 18 hepatectomías derechas extendidas (HDE, 29\%), 14 hepatectomías izquierdas (HI, $22,6 \%$ y 7 hepatectomías izquierdas extendidas (HIE, 11,3\%). En 4 pacientes con MHCCR se realizó en forma sincrónica la cirugía resectiva del primario colorrectal. La vía de abordaje más utilizada fue por laparotomía en 59 pacientes $(95,2 \%)$ y sólo 3 sometidos a resección laparoscópica $(4,8 \%)$.

Al comparar las características biodemográficas y clínicas preoperatorias de ambos grupos, no se encontraron diferencias significativas, lo que se detalla en la Tabla 1. La Tabla 2 muestra las indicaciones de las RHM sin diferencias significativas entre los grupos A y B.

Cuando se analizó el tipo de RHM y los tiempos operatorios de ambos grupos, no se observó ninguna diferencia, sin embargo, el tiempo de duración de la MP fue más prolongada en el grupo que recibió esteroides (43 $\pm 3,3$ vs $27 \pm 2,1 \mathrm{~min}, \mathrm{p}<0,05)$. Respecto al sangrado intra-operatorio, se observó que la mediana de sangrado en ambos grupos fue de $800 \mathrm{ml}(\mathrm{p}=0,512)$ sin diferencias en el número de unidades de glóbulos rojos transfundidos (grupo A: $2(0-6)$ vs grupo B: $0(0-4), \mathrm{p}=0,21)$, ni en el hematocrito de control posoperatorio (grupo A: $32,4 \%$ vs grupo B: $31,5 \%, p=0,57)$. La alteración en las pruebas hepáticas de ambos grupos se detalla en la Tabla 3.

La Figura 2 muestra el impacto del DIR según alteración de pruebas hepáticas. Se observó que los pacientes sometidos a RHM con MP que recibieron MPD presentaron una menor elevación de las fosfatasas alcalinas (FA) en los días 1 (75 (30-233) U/L vs $98(46-655) \mathrm{U} / \mathrm{L}, \mathrm{p}=0,034)$ y $5(124,5(41,4)$ $\mathrm{U} / \mathrm{L}$ vs $178,2(80,7) \mathrm{U} / \mathrm{L}, \mathrm{p}=0,005)$ en comparación con aquellos sin MPD. También se observó una menor elevación de la bilirrubina total (BT) en el día $1(1,29(0,35-10,37) \mathrm{mg} / \mathrm{dl}$ vs $2,01(0,3-20,76)$ $\mathrm{mg} / \mathrm{dl}, \mathrm{p}=0,042)$; de la bilirrubina directa (BD) al día $5(0,73(0,11-10,93) \mathrm{mg} / \mathrm{dl}$ vs $1,19(0,08-8,75)$ $\mathrm{mg} / \mathrm{dl}, \mathrm{p}=0,018)$. También se observó que la BT al momento del alta fue menor en los pacientes que recibieron MPD $(0,84(0,25-18,35) \mathrm{mg} / \mathrm{dl}$ vs 1,42 $(0,4-19,86) \mathrm{mg} / \mathrm{dl}, \mathrm{p}=0,046)$. Cuando se comparó la magnitud de citolisis según alteración de aminotransferasas y la alteración de los factores de coagulación según el tiempo de protrombina, no se observó diferencias significativas entre ambos grupos (Figura 3). Tampoco hubo diferencias respecto al alza máxima de GOT y GPT. 
Tabla 3. Descripción de los tipos de resección hepática mayor, variables intraoperatorias y posoperatorias

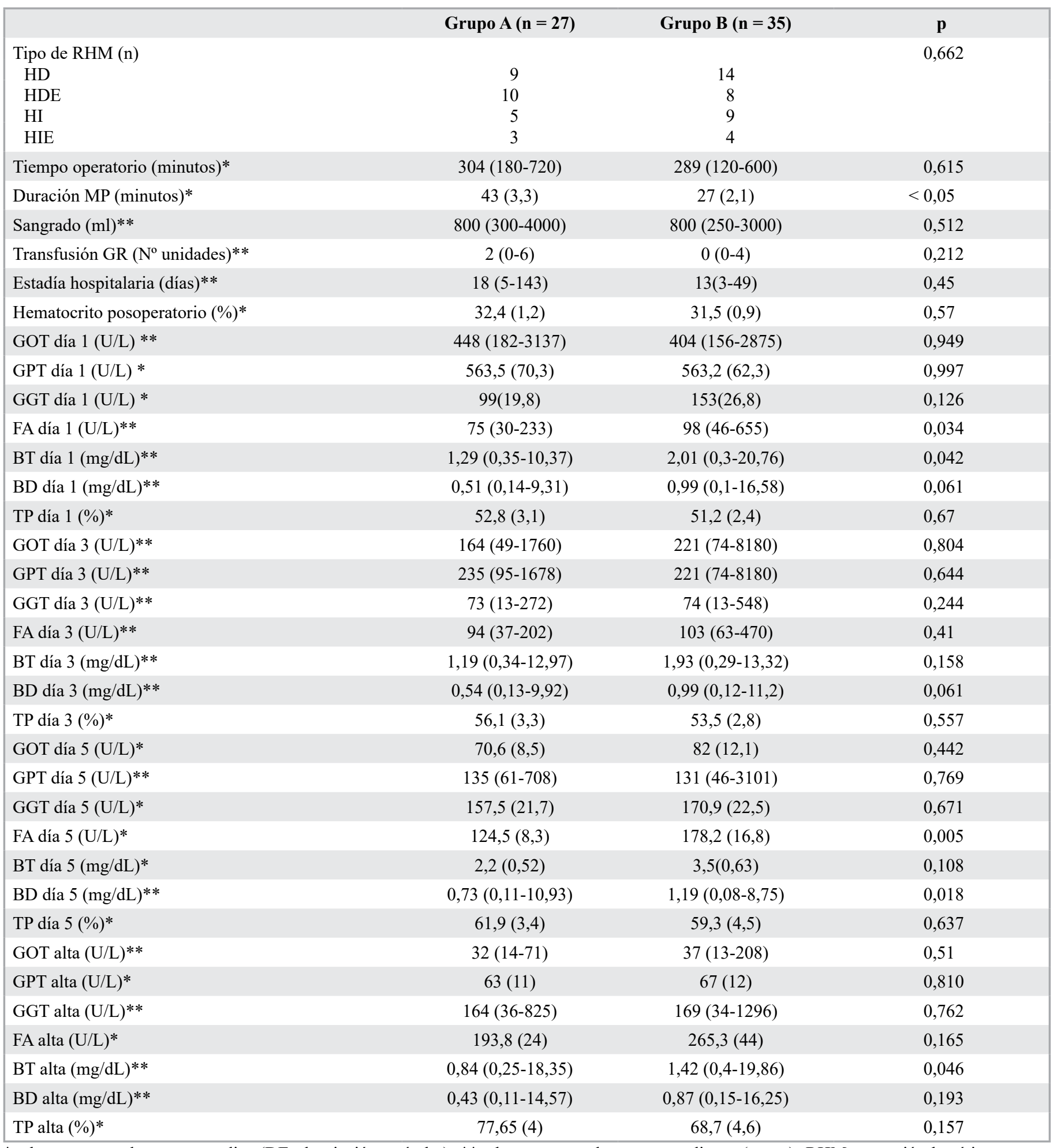

*valores expresados como medias (DE: desviación estándar); **valores expresados como medianas (rango); RHM: resección hepática mayor; HD: hepatectomía derecha; HDE: hepatectomía derecha extendida; HI: hepatectomía izquierda; HIE: hepatectomía izquierda extendida; MP: maniobra de Pringle; GR: glóbulos rojos. 


\section{ARTíCULO ORIGINAL}
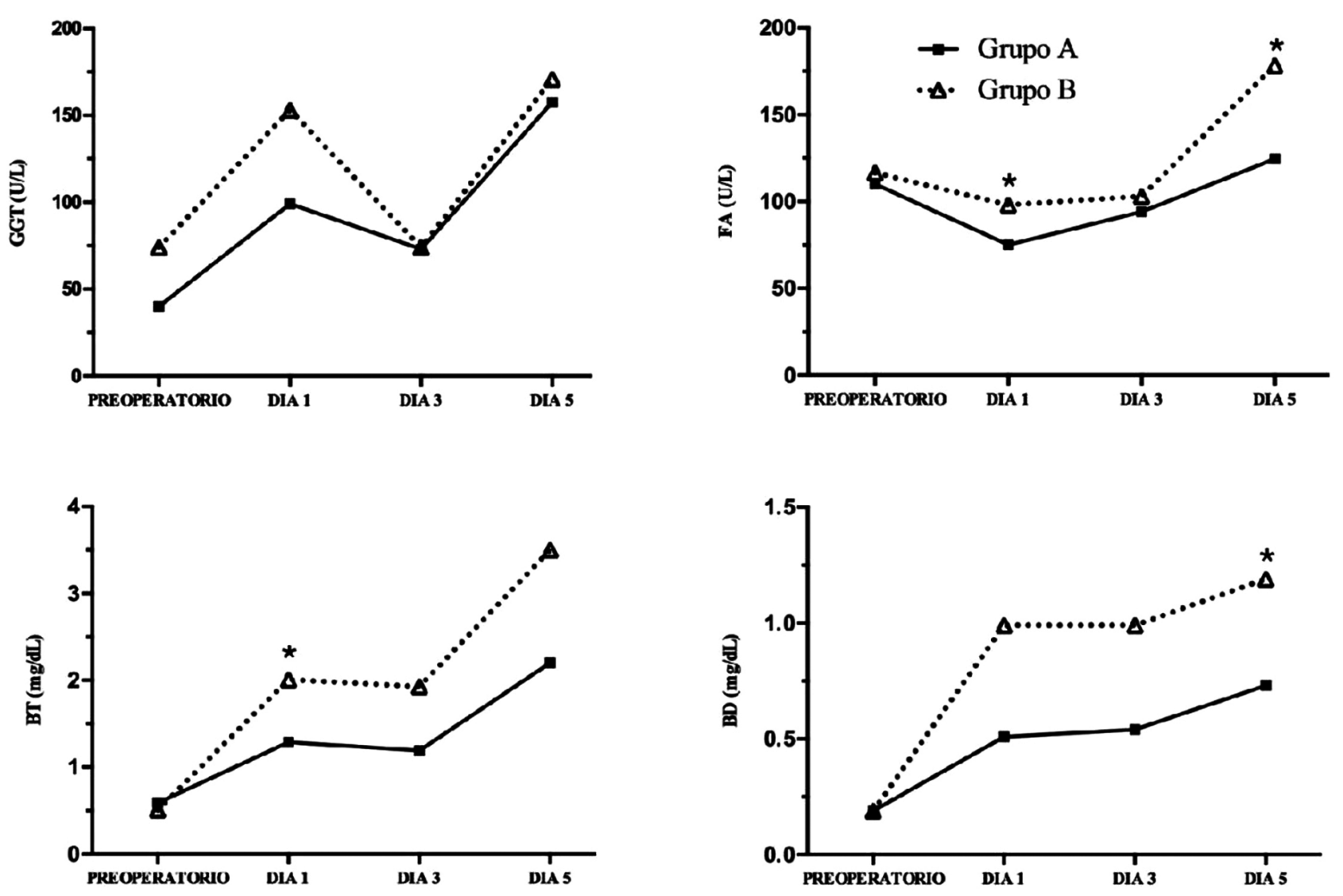

Figura 2. Cambios posoperatorios de la GGT, FA, BT y BD al día 1,3 y 5 entre el grupo que recibió metilprednisolona (grupo A, línea continua) y grupo que no recibió esteroides (grupo B, línea discontinua). Asteriscos representan $p<0,05$.
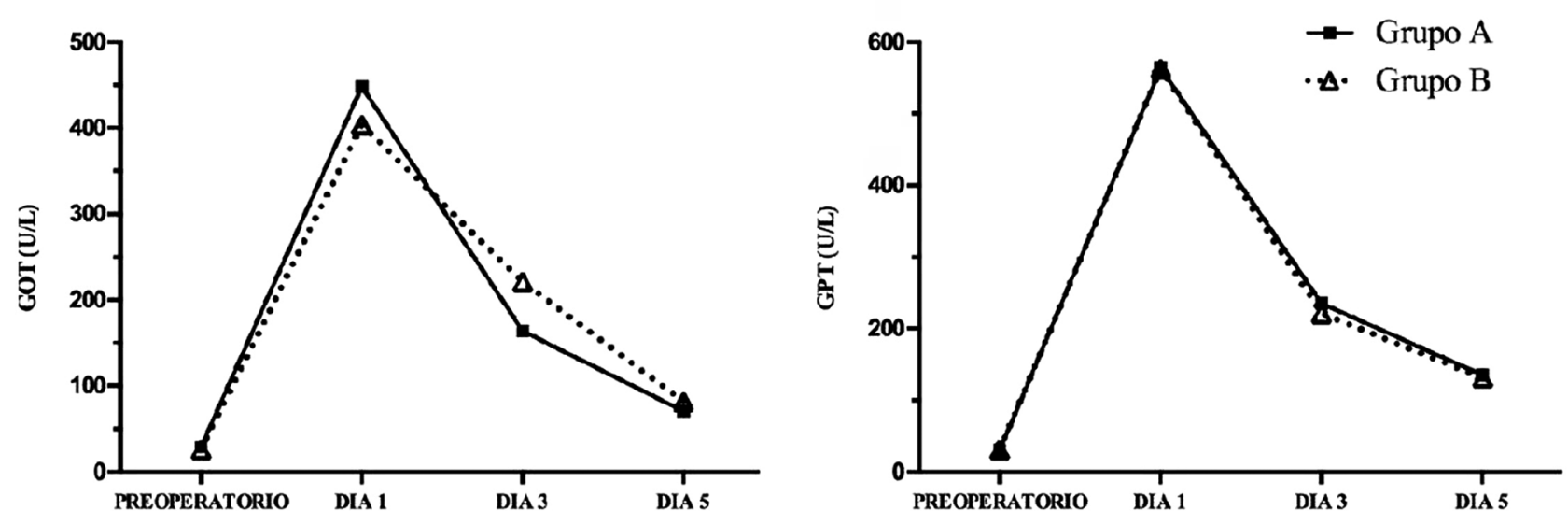

Figura 3. Representación del grado de citolisis basándose en la medición de GOT y GPT al día 1,3 y 5 entre el grupo que recibió metilprednisolona (grupo A, línea continua) y grupo que no recibió esteroides (grupo B, línea discontinua). 
Tabla 4. Complicaciones según grupo

\begin{tabular}{|lcc|}
\hline & $\begin{array}{c}\text { Grupo A } \\
(\mathbf{n = 2 7 )}\end{array}$ & $\begin{array}{c}\text { Grupo B } \\
(\mathbf{n = 3 5})\end{array}$ \\
\hline Sin complicación & 14 & 15 \\
\hline Clavien I & 2 & 3 \\
Clavien II & 3 & 8 \\
Clavien IIIa & 1 & 0 \\
Clavien IIIb & 3 & 3 \\
Clavien IVa & 0 & 3 \\
\hline Clavien IVb & 2 & 0 \\
Clavien V & 0 & 1 \\
\hline p = 0,285. & & \\
\hline
\end{tabular}

Un $51,6 \%$ de los pacientes presentaron 1 o más complicaciones; $19,4 \%$ del tipo III y IV según Dindo-Clavien. La morbilidad en ambos grupos se detalla en la Tabla 4. Respecto a la morbilidad específica (Tabla 4), se dividió las complicaciones en infecciosas, hemorrágicas, tromboembólicas, biliares y otras, sin observarse diferencias significativas entre ambos grupos $(p=0,97)$. Un paciente $(1,6 \%)$ sometido a una RHM extendida simultánea a una cirugía de resección colorrectal que no recibió esteroides falleció como consecuencia de una trombosis porto-mesentérica a los 14 días del posoperatorio. Respecto a los 4 pacientes con cirugía sincrónica hepática y colorrectal, sólo uno recibió esteroide previo a la realización de la MP.

\section{Discusión}

Los esteroides en dosis estándar han sido utilizados durante el preoperatorio para disminuir la respuesta al estrés quirúrgico y el DIR en pacientes sometidos a cirugía hepática, debido a que reducen la apoptosis y disrupción parenquimatosa en un hígado isquémico ${ }^{6,7}$. Si bien algunos metaanálisis muestran que mejora la morbilidad, reduce las complicaciones y mejora el perfil hepático posoperatorio, los trabajos analizados son heterogéneos e incluyen un bajo número de pacientes sometidos a $\mathrm{RHM}^{8,9}$. El presente estudio, a diferencia de publicaciones anteriores, estudia el efecto de los esteroides en dosis bajas administrados en el intraoperatorio en pacientes sometidos a RHM bajo MP.

Un metaanálisis australiano concluyó que existe una reducción significativa en los niveles de IL-6 y BT en el primer día posoperatorio en el grupo que utilizó esteroides en dosis estándar, sin otras diferencias significativas ${ }^{9}$. En nuestra serie, si bien no se realizó medición de citoquinas, se observó que los pacientes que recibieron esteroides en dosis bajas (< a $500 \mathrm{mg}$ de MPD) presentaron menor elevación de la BT al día 1, BD al día 5 y BT al alta, además de una menor elevación de las FA al día 1 y en el día 5, lo que corrobora que los esteroides tienen un efecto protector del DIR. Si bien el mecanismo que explica este efecto beneficioso no está del todo claro, se postula que estaría relacionado con la supresión de las citoquinas inflamatorias, aumento del flujo sanguíneo, estabilización de la membrana celular y disminución de las proteasas lisosomales ${ }^{9}$. También se ha atribuido, a una reducción de la respuesta inflamatoria al inducir la expresión de citoquinas antiinflamatorias como la IL-10 10,11 , inhibidoras de la producción intracelular de radicales libres del oxígeno ${ }^{12}$. Algunos autores desaconsejan el uso de la MPD, argumentando la existencia de un potencial efecto deletéreo de los esteroides sobre la regeneración hepática, al disminuir los niveles de IL-6; sin embargo, se sabe que una sobreproducción de IL-6 también podría inhibir la reparación hepática $^{13}$. Un estudio en animales demostró que no existe efecto negativo de los esteroides en la regeneración hepática lo que apoya la utilización segura de éstos ${ }^{14}$. En nuestra serie, no se encontró diferencias significativas en cuanto a sangrado, complicaciones posoperatorias, estadía hospitalaria ni mortalidad entre ambos grupos, por lo que, hipotéticamente los esteroides en dosis bajas tendrían un perfil de efectividad y seguridad adecuados.

Actualmente, el esteroide más utilizado es la MPD intravenosa por tener un tiempo de acción prolongado, efecto antiinflamatorio más potente que el cortisol y menor impacto sobre los electrolitos plasmáticos 9 . Se usa, por lo general, en dosis altas, en promedio aproximado de $500 \mathrm{mg}$, habiéndose descrito incluso hasta $30 \mathrm{mg} / \mathrm{kg}$ en algunas series ${ }^{9}$. En su mayoría, son utilizados previos a la cirugía dado que se sabe que son menos efectivos si se dan luego de la inducción de la respuesta inflamatoria ya que el efecto antiinflamatorio aparece 1-2 horas después de su administración ${ }^{15}$. Nuestro estudio muestra, sin embargo, que la utilización de esteroides intraoperatorios tiene un efecto similar al de su administración preoperatoria. Respecto al uso en dosis menores, Sayama et al mostraron que el uso de $250 \mathrm{mg}$ de MPD en el preoperatorio redujo la producción posoperatoria de IL-6 y factor estimulante de colonia de granulocitos en pacientes sometidos a cirugía esofágica por cáncer ${ }^{16}$. Aún no hemos encontrado estudios publicados con estas dosis en RHM. En cuanto a la relación conocida entre el uso de esteroides y el desarrollo de complicaciones 
infecciosas, nuestro estudio no muestra diferencias respecto al desarrollo de infecciones. Cabe destacar, que nuestra serie tuvo mortalidad de un paciente del grupo que no recibió esteroides, a causa de una trombosis portal y mesentérica. Creemos que esta complicación estuvo relacionada con la combinación de intervenciones complejas (colectomía extendida + hepatectomía mayor) realizadas en el mismo tiempo quirúrgico y no específicamente con el tipo de RHM. Actualmente, hemos modificado nuestra conducta respecto de las resecciones simultáneas del tumor primario con RHM por metástasis hepáticas, hacia criterios más seguros según las magnitudes de resección requeridas en el tratamiento de pacientes con MHCCR.

Nuestros hallazgos permiten concluir que la utilización de dosis bajas de MPD disminuye el impacto del DIR relacionado a la utilización de MP en RHM sin los efectos secundarios de la administración de esteroides en dosis altas. Sin embargo, la debilidad de este estudio reside en su carácter retrospectivo y observacional. Es necesario obtener un nivel más elevado de evidencia, con diseños de estudios prospectivos y aleatorizados, idealmente multicéntricos, que confirmen estos resultados.

\section{Responsabilidades éticas}

Protección de personas y animales. Los autores declaran que para esta investigación no se han realizado experimentos en seres humanos ni en animales.

Confidencialidad de los datos. Los autores declaran que han seguido los protocolos de su centro de trabajo sobre la publicación de datos de pacientes.

Derecho a la privacidad y consentimiento informado. Los autores declaran que en este artículo no aparecen datos de pacientes.

\section{Referencias}

1. Lafaro K, Buettner S, Maqsood H, Wagner D, Bagante F, Spolverato G, et al. Defining Post Hepatectomy Liver Insufficiency: Where do We stand? J Gastrointest Surg. 2015;19: 2079. https:// doi.org/10.1007/s11605-015-2872-6.

2. Balzan S, Belghiti J, Farges O, Ogata S, Sauvanet A, Delefosse D, et al. The "50-50 criteria" on postoperative day 5: an accurate predictor of liver failure and death after hepatectomy. Ann Surg. 2005;242:824-8. doi: 10.1097/01. sla.0000189131.90876.9e

3. Van Den Broek MAJ, Olde Damink SWM, Dejong CHC, Lang H, Malagó $\mathrm{M}$, Jalan R, et al. Liver failure after partial hepatic resection: definition, pathophysiology, risk factors and treatment. Liver International 2008;28:767-80. doi:10.1111/j.14783231.2008.01777.x

4. Dindo D, Demartines N, Clavien PA. Classification of Surgical Complications: A New Proposal With Evaluation in a Cohort of 6336 Patients and Results of a Survey. Annals of Surgery 2004;240:205-13. doi:10.1097/01. sla.0000133083.54934.ae.

5. Clavien PA, Barkun J, de Oliveira ML, Vauthey IN, Dindo D, Schulick RD, et al. The Clavien-Dindo classification of surgical complications: five-year experience. Ann Surg. 2009;250:187-96. doi: 10.1097/SLA.0b013e3181b13ca2.

6. Saidi RF, Chang J, Verb S, Brooks S, Nalbantoglu I, Adsay V, et al. The effect of methylprednisolone on warms pischemiareperfusion injury in the liver. Am J Surg. 2007;193: 345-7. doi: 10.1016/j. amjsurg.2006.09.017.

7. Figueroa I, Santiago-Delpin EA. Steroid protection of the liver during experimental ischemia. Surg Gynecol Obstet. 1975;140:368-70.

8. Orci LA, Toso C, Mentha G, Morel P, Majno PE. Systematic review and metaanalysis of the effect of perioperative steroids on ischaemia-reperfusion injury and surgical stress response in patients undergoing liver resection. Br J Surg. 2013;100:600-9 .doi: 10.1002/bjs.9035.

9. Richardson A, Laurence JM, Lam VW. Use of pre-operative steroids in liver resection: a systematic review and metaanalysis. HPB (Oxford) 2014;16:12-9.doi: 10.1111/hpb.12066.

10. Aldrighetti L, Pulitanò C, Arru M, Finazzi $\mathrm{R}$, Catena M, Soldini L, et al. Impact of preoperative ischemia-reperfusion injury and systemic responses in liver surgery: A prospective randomized study. Liver Transplant 2006;12:941-9. doi: 10.1002/lt.20745.

11. Yoshidome H, Kato A, Edwards MJ, Lentsch AB. Interleukin-10 suppresses hepatic ischemia/reperfusion injury in mice: implication of a central role for nuclear factor $\mathrm{kb}$. Hepatology 1999;30:203-8. doi:10.1002/ hep.510300120.

12. Barnes PJ. Anti-inflammatory actions of glucocorticoids: molecular mechanisms. Clin Sci. 1998;94:557-72. doi: 10.1042/ cs0940557.

13. Wustefeld T, Rakemann T, Kubicka S, Manns MP, Trautwein C. Hyperstimulation with interleukin 6 inhibits cell cycle progression after hepatectomy in mice. Hepatology 2000;32:514322. doi: 10.1053/ jhep.2000.16604.

14. Glanemann M, Strenziok R, Kuntze R, Munchow S, Dikopoulos N, Lippek $\mathrm{F}$, et al. Ischemic preconditioning and methylprednisolone both equally reduce hepatic ischemia/reperfusion injury. Surgery 2004;135:203-14. doi. org/10.1016/j.surg.2003.08.011.

15. Holte K, Kehlet H. Perioperative singledose glucocorticoid administration: pathophysiologic effects and clinical implications. J Am Coll Surg. 2002;195:694-71.

16. Sayama J, Shineha R, Yokota K, Hirayama $\mathrm{K}$, Higuchi N, Ohe H, et al. Control of the excessive reaction after surgery for esophageal cancer with preoperative administration of the corticosteroids. J Gastroenterol Surg. 1994;27: 841-8. doi. org/10.5833/jjgs.27.841. 